COMMENT. Previous studies have shown that infants born very preterm (VPT), $<33$ weeks gestation and of low birth weight $(<2500 \mathrm{~g})$, are at risk of hemorrhage and hypoxicischemic damage that results in dilated ventricles, loss of white matter, and enlarged subarachnoid space (Volpe JJ. Pediatrics 2003;112:176-180); (Inder TE et al. J Pediatr 2003;143:171-179). Subarachnoid fluid collections in very low birth weight infants, sometimes called "external hydrocephalus," may be associated with transient macrocrania and neurodevelopmental abnormalities (hypertonia and hyperreflexia) that resolve by 18 months of age. (Al-Saedi SA et al. J Pediatr 1996;128:234-236). Half of VPT adolescents show persisting brain abnormalities, with smaller cortical volumes and larger lateral ventricles compared to controls. (Cooke RW et al. Arch Dis Child Fetal Neonatal Ed 1999;81 (F):116-121).

Computational morphometry used to process MRI data in the present study identifies focal localized changes in GM and WM concentration. The findings suggest that alterations in grey and white matter volume demonstrated in temporal, frontai, cerebellar and other regions of brain may be responsible for the cognitive impairments found in adolescents born VPT. The cerebral developmental changes following VPT birth result not only in GM and WM loss, but also in cortical and subcortical tissue excesses, often in adjacent and structurally associated areas. Infants who experience the greatest degree of perinatal insult exhibit the most severe GM and WM alterations and have the highest risk of neurodevelopmental compromise. When VPT birth is complicated by severe brain injury, brain plasticity compensates for resulting cell loss, with production of extra cells and synapses that normally become 'pruned' during later development. These compensatory processes are particularly extensive in preterm infants showing the most severe neonatal ultrasound abnormalities.

Selective vulnerability varies with the stage of development of different brain regions. GM volume in frontal lobes increases during preadolescence, the prefrontal areas reaching full maturity in late adolescence. Temporal lobe GM development peaks in mid-adolescence. VPT children may show not only a global delay in brain maturation but also, differences in GM and WM volumes. Identification of these brain volume changes by MRI in early life may be used as a clinical marker of increased risk of cognitive impairment at a later age, and may lead to educational intervention.

\title{
ETIOLOGY AND TREATMENT OF DEVELOPMENTAL STAMMERING
}

The etiology and treatment of developmental stammering in childhood (DS, also called idiopathic stammering or stuttering) are reviewed by a speech pathologist and psychologist at the University of Reading, UK. Prevalence is estimated at 1 to $3 \%$ of the population. DS is distinguished from neurogenic stammering (secondary to stroke, tumor, or degenerative disease) and psychogenic stammering. DS usually develops in pre-school age groups, the mean age at onset of 4 years, with $75 \%$ cases beginning before age 6 . The cause is multifactorial, demands placed on the child exceeding the capacity to manage speech and language variables. The evidence for a genetic component to stammering is strong, twin studies showing concordance in monozygotic twins of $75-89 \%$. Some children with DS are linguistically advanced while others are delayed. A mismatch between motor speech and language abilities may cause impairment in fluency, when articulation skills are not 
sufficiently developed to keep pace with verbal output. Another theory invokes a faulty auditory processing, that may respond to delayed auditory feedback therapy. Neuroimaging in adults who stammer may show hemispheric asymmetries, and neurochemical studies report both increased and decreased levels of dopamine. Risperidone, a D2 antagonist, may reduce severity of stammering in some adults. Treatment recommendations in children vary from an indirect approach, with changes in the child's environment to reduce demands, to direct intervention, targeting speech output and capacity. Complete recovery, with or without therapy, is common before adolescence. Approximately $74 \%$ of children who stammer recover, $89 \%$ of young females. Boys are affected most frequently; those with a late onset of DS have the poorest prognosis, especially when complicated by speech and language delay. (Ward D. The aetiology and treatment of developmental stammering in childhood. Arch Dis Child January 2008;93:68-71). (Respond: Dr David Ward, School of Psychology and Clinical Language Sciences, University of Reading, Reading RG6 6AL, UK).

COMMENT. Stammering is developmental (idiopathic) or acquired. Particularly in adults, it may be secondary to organic brain disease or of psychogenic origin. In children it is most likely developmental and usually transient, requiring only an adjustment of parental handling or school pressure. If persistent and complicated by seizures or other neurologic manifestations, investigations should include an EEG and MRI. In rare cases, the differential diagnosis may include Landau-Kleffner syndrome; symptoms are associated with an acute onset of loss of speech comprehension and auditory agnosia, seizures and/or a paroxysmal EEG with bitemporal discharges. (Morrell F et al. Brain 1995;118:1529-1546).

Structural and functional abnormalities of the motor system in developmental stuttering are reported by researchers at the Departments of Experimental Psychology and Clinical Neurology, University of Oxford; and Department of Psychology, University College London, UK. (Watkins KE, Smith SM, Davis S, Howell P. Brain January 2008;131(1):50-59). Using functional and diffusion imaging, motor and language areas were examined in brains of 12 young subjects (aged 14-27 years;avg 18 years; 1 left-hander) who stutter. During speech production, people who stutter show overactivity relative to controls in the anterior insula, cerebellum and midbrain bilaterally and underactivity in the ventral premotor, Rolandic opercular and sensorimotor cortex bilaterally and Heschl's gyrus in the left hemisphere. The overactivity in the midbrain, at the level of the substantia nigra, red and subthalamic nuclei, is consistent with a previous report of excess dopamine in adults who stutter. Areas with underactivity are associated with articulation and speech production, and show loss of white matter. Stuttering is related to disruption in neural systems that support the execution of fluent speech.

\section{BRAINSTEM MALFORMATIONS}

Malformations of the brainstem in 138 patients identified aver a 10 year period are classified according to MRI findings and by embryological cause in a study at University of California at San Francisco, and University of Chicago, IL. The pons was involved in 114, midbrain in 45 , and medulla in 14. More than 1 region was affected in 53 patients. Malformations were classified in four groups: 1) disorder of brainstem segmentation or induction; 2) segmental hypoplasia; 3) postsegmentation malformation (associated with migration abnormalities); and 4) abnormal cortical organization. Segmentation anomalies 